Wie schon vorher angedeutet, erfordert der Dienstbetrieb dieser Stelle, soll sie ihre Aufgabe richtig und segensreich ausüben, daß in ihr nur genügend erfahrene Chemiker und Ingenieure tätig sind. Diesen müssen aber auch zum Beraten in juristischen Fragen geeignete Rechtskundige und zum Nachprüfen besonders umfangreicher und schwieriger Bilanzen, wozu dem Techniker gewöhnlich die Zeit fehlt, auch bilanztechnisch erprobtes Personal zur Seite stehen. An einem Grundsatz muß aber unbedingt festgehalten werden, die eigentliche Prüfung darf und muß einzig und allein vom Techniker, gleich ob Chemiker oder Ingenieur, vorgenommen werden, denn nur diese beiden sind allein dazu geeignet, und hier muß genau so, wie seinerzeit für die Beschaffungsstellen (siehe diese Zeitschrift, wirtsch. Teil 1920, $\mathrm{S} .65$ ) ausgeführt und gefordert, mit dem Wahne endgültig gebrochen werden, daß das Hineinberufen in eine derartige Stelle genügt, um mit einem Schlage die nötigen Kenntnisse und Erfahrungen hervorzuzaubern. Es ist daher ebenso grundfalsch, diese Posten dem reinen Juristen, der nicht gleichzeitig über eine jahrzehntelange Erfahrung als Vorstand chemischer oder anderer industrieller Werke verfügt, also industriell erfahren ist, oder auch Bücherrevisoren $\mathrm{zu}$ überweisen, denn diese müssen immer versagen, da ihnen die nötige und unerläßliche technische Erfahrung und der sichere Blick und das Verständnis für technische Fragen fehlt. Gerade der Ernst der Zeit, die bittere Not des Vaterlandes zwingen uns ebenso, wie der uns auferlegte Schmachfriede, mit allem Alten, Ửberlebten zu brechen, vollkommen frisch und sachgemäß wieder neu aufzubauen, denn nur so kann Deutschland wieder bei äußesrster Anspannung aller produktiven Kräfte auf eine Wiedergesundung und Erstarkung hoffen, und dazu gehört in erster Linie das Fortbestehen einer Preisprüfungsstelle für die Beschaffungen des Heeres usw. in den geschilderten Abmessungen und Zusammensetzungen. Eine derartige amtliche Stelle wird für den unaufschiebbaren Preisabbau, ohne den eine Wiedererholung Deutschlands und Gesundung der Verhältnisse ausgeschlossen ist, höchst segensreich wirken. Ihr müssen, wie es zuletzt im Kriege erforderlich war, alle Abschlüsse zur Prüfung und Genehmigung vorgelegt werden, nur damn kann Wucher und Ausbeutung möglichst verhindert werden. Wäre, um nur ein Beispiel anzuführen, jener Vertrag über Lieferung cines sehr großen Postens Uniformtuch für die grüne Sicherheitspolizei einer derartigen Stelle vorgelegt worden, es hätte sich kein Fachmann gefunden, der ihn gebilligt hätte. Jeder Eingeweihte mußte wissen, daß derartige Tuche wesentlich billiger und besser unsere hochentwickelte Tuchindustrie herzustellen in der Lage war, er hätte uns vor der traurigen Erfahrung bewahrt, daß wir unseren ärgsten Feinden abgängiges Material, das überdies noch umgefärbt werden mußte, zu Phantasiepreisen abnahmen, unsere Valuta unnütz schädigten und die Arbeitslosigkeit in der Textilindustrie vermehrten und anstatt Hunderten von Arbeiterfamilien anf Monate sicheres Brot zu gewähren, sie durch Stillegen der Betriebe auf die Straße setzten. Das ist aus vaterländischen Gründen und im Sinne einer volkswirtschaftlich gesunden Auftragserteilung aufs schärfste zu verurteilen, zumal die Befürchtung sehr nahe liegt, daß außer diesem einen zufällig bekannt gewordenen Musterbeispiele noch viele andere ähnliche Vorkommnisse vorhanden sind.

Diese nicht länger mehr aufschiebbare Frage vor Ihnen aufzurollen und zu weiterer Behandlung anzuregen, ist Zweck dieser Worte.

[A. 216.]

\section{Der Wert der Stockschen Nomenklatur für den Chemieunterricht nebst Bemerkungen zur Namengebung der Element-Symbole.}

\author{
Von Prof. O. OHmann, Berlin.
}

(Eingeg. am 12./11. 1920.)

Die von Prof. A. S t o c k in dieser Zeitschrift (32, S. 373 f. [1919]) aufgestellte Namengebung, die bei den mit verschiedener Wertigkeit auftretenden chemischen Elementen diese Wertigkeit im Namen der Verbindung zum Ausdruck bringt, kann sich als ersten Erfolg buchen, daß sie in dem ausgezeichneten ,Lehrbuch der anorganischen Chemie $^{i 6}$ von $\mathbf{K}$ a r l A. H of $\mathrm{mann}$ (Braunschweig, Vieweg und Sohn) bereits zur Anwendung gelangt ist. Die nachfolgenden Zeilen haben den Hauptzweck, darzulegen, da $B$ diese Namengebung sich auch für den gesamten Unterricht, insbesondere für den Chemieunterricht an den höheren Lehranstalten sehr gut eignet. Für den letzteren handelt es sich zunächst um eine möglichst einfache und klare Ausdrucksweise, um Eindeutigkeit bei den Benennungen der Verbindungen. Diese Eigenschaften finden wir in den St ockschen Bezeichnungen verwirklicht. Die Benennungen Eisen(3)-Chlorid, Kupfer(1)-Oxyd usw. sind völlig durchsichtig und haben vor den bisherigen Namen Ferrichlorid, Cuprooyxd usw. entschiedene Vorzüge; ebenso bei den Sauerstoffsalzen Eisen(2)-Sulfat usw. gegenüber Ferrosulfat oder schwefelsaurem Eisenoxydul usw. Die Benennungen Ferro-, Ferri- usw. bedeuteten s. Z. wohl einen erheblichen Fortschritt, zeigen aber andererseits bei näherer Betrachtung und Vergleichung beträchtliche Unstimmigkeiten. Die Endungen -o und -i haben insofern etwas Vages an sich, als sie sich auf ganz verschiedenartige Wertigkeiten beziehen, bald auf ein 1- und 2-wertiges Element (Cupro-, Cupri-), bald auf ein 2- und 3-wertiges (Ferro-, Ferri-) bald auf ein 2- und 4-wertiges (Stanno-, Stanni) usw. Im Unterricht muß daher bei jedem Element erst festgesetzt werden, auf was für Wertigkeiten sich die Endungen o- und i- beziehen. Diese Unbestimmtheiten und Umwege fallen bei der $\mathrm{St}$ o c $\mathrm{k}$ schen Namengebung gänzlich fort, die Benennungen Kupfer(2)- oder Kupfer(1) Oxyd usw. bringen die Natur der betreffenden Verbindung klar und unzweideutig zum Ausdruck und führen entweder unmittelbar oder auf ganz leichte Weise zur Aufstellung der zugehörigen FormeJ. Dies alles macht die neue Bezeichnungsweise für den Unterrichtsgebrauch geeignet. Hierbei wird es nützlich sein - zumal gegenüber den griechischen, die Atom $\mathbf{z}$ a $\mathbf{h}$ l des $\mathrm{n}$ a $\mathrm{c} h$ folgenden Flementes betreffenden Vorsatzsilben di-, tri- usw. - auch das Negative rech deutlich hervorzuheben und etwa zu sagen: ,Die eingeklammerte Zahl gibt also immer die Wertigkeit des davorstehenden Eiementes an, sie hat mit irgend einer A tom $\mathrm{zahl} \mathrm{nichts} z u$ t u n" wie ich es in der soeben erschienenen Neuauflage meines ,Leitfaden der Chemie" (Berlin, Winckelmann u. Söhne) ausgedrückt habe Vielleicht schleifen sich später die oben angeführten Namen Eisen(3) Chlorid usw. noch ein wenig in Eisen(3)chlorid usw. ab.

Für den einführenden Chemieunterricht handelt es sich aber auch noch um eine möglichst volkstümliche Bezeichnungsweise, wie sie sich in den Namen chlorsaures Kalium, doppeltkohlensaure Natrium usw. ausspricht. Die wichtigen Gruppennamen der Salze, die Nitrate, Sulfate usw. sollen gewiß nicht unerwähnt bleiben, haben aber für den Anfang etwas Fremdartiges, fast Abstoßendes, und dürfen daher nicht zuerst geboten werden; alle wissenschaftliche Bezeichnungsweise $\mathrm{muB}$ im Unterricht vielmehr erst aus den volks tümlichen deutschen Namen herauswachsen, damit der Schüle wirklich Liebe zu dem neuen, nicht leicht zugänglichen Lehrgegen stand gewinnt. Es ist nun ein gutes Zeichen, daß sich die $\mathrm{S} \mathrm{t} \mathrm{o} \mathrm{c} \mathrm{k}$ sche Bezeichnungswelse dieser Forderung leicht anpassen läßt. Wo nämlich verschiedenerlei Wertigkeit vorliegt, z. B. bei den beiden Eisonsulfaten, ergeben sich leicht die Benennungen ,schwefelsaures Eisen $(2)^{6 "}$ und ,,schwefelsaures Eisen $(3)^{\prime \prime}$, ebenso , salpetersaures Quecksilber(1)" usw.; ebenso kann man allgemein statt Ferrosalze Eisen(2)-salze, statt Cuproverbindungen Kupfer(1)verbindungen usw. setzen. Diese natürlichen Folgerungen haben, wie aus einer mündlichen Äußerung hervorgeht, bereits die volle Zustimmung des Urhebers der neuen Bezeichnungsweise gefunden. So fördert die $\mathrm{S} t$ o c $\mathrm{k}$ sche Namengebung auch das wichtige volkstümliche Moment im chemischen Unterricht der höheren Lehranstalten und aller Fach schulen. - Nicht minder sind seitens dieses Unterrichts die weiteren Vorschläge S t o c ks zu begrüßen, -carbid und -hydrid statt.carbure und hydrüre zu setzen, sowie den Ausdruck -peroxyd auf die wirklich Wasserstoffperoxyd liefernden Metalloxyde zu beschränken und nicht für die Oxyde 4-wertiger Metalle, wie $\mathrm{MnO}_{2}, \mathrm{PbO}_{2}$ gelten zu lassen.

Die $\mathrm{St}$ o c k schen Vorschläge werden nicht verfehlen, mannigfache Anregungen zu geben. Zunächst habe ich neulich in einem Aufsatz ,Zur Weiterentwicklung der chemischen Namengebung“" (Z. f. d. phys. u. chem. Unt. 33, S. 41 ff. [1920]) in Anknüpfung an jene Vorschläge einige Wünsche zum Ausdruck gebracht, z. B. man möchte, analog der Benennung Ammonsalpeter, die sich im Kriege bei den Pionieren völlig eingebürgert hatte, auch von Ammonhydroxyd und allgemein nur von Ammon reden und schreiben; die schleppende lateinische Endigung ist wirklich überflüssig. Letztere kann auch beim langatmigen Aluminium fortfallen, Aluminmetall usw. hat guten Klang; ähnlich Plastilin statt Plastilina. Aüch wurde dort, gestützt auf Autoritäten, dargelegt, da ß man Barium (statt Baryum) schreiben müsse, desgleichen auf das Unstatthafte der Schreibweise Silizium und Kalzium hingewiesen.

Heute mögen noch einige Bemerkungen zur Nomenklatur der Elementsymbole gestattet sein. Diese läßt sich auf fünf Regeln zurückführen. Die Abkürzungen entsprechen' 1) dem Anfangsbuchstaben des internationalen latinisierten Namens $(\mathbf{H}, \mathrm{C}, \mathrm{N}$ usw.) oder es tritt zu diesem 2. der unmittelbar benachbarte Buchstabe ( $\mathrm{Si}, \mathrm{Br}$ usw.) oder 3. der nächste Konsonant (Mg, Mn usw.) oder auch 4. der Anfangsbuchstabe der zweiten Silbe ( $\mathrm{Pb}, \mathrm{Cs}, \mathrm{Sb}$ usw.) oder endlich 5. irgend ein anderer kennzeichnender Laut (Cl, Pd usw.). Treten nun viel Elemente mit gleichen Anfangsbuchstaben auf, so zeigt die bisherige Bezeichnung einige Inkonsequenzen, die leicht zu Verwechslungen Anlaß geben können. So gibt es sieben Elemente, die mit $\mathrm{T}$ beginnen: Tantal $=\mathrm{Ta}$, Tellur $=\mathrm{Te}$, Terbium $=\mathrm{Tb}$, Titan $=\mathrm{Ti}$, Thulium $=\mathrm{Tu}$, Thallium $=\mathrm{Tl}$, Thorium $=\mathrm{Th}-$ drei davon fangen mit Th an, das Symbol Th ist demnach dreideutig, und es ist wenig gerechtfertigt, es für Thorium zu verwenden. Folgerichtiger wäre, hier Regel 4 oder 5 anzuwenden; was dem Thulium ( $=\mathrm{Tu}$ ) recht ist, sollte dem Thorium billig sein, d. h. man sollte Th or i $m=$ T o setzen. Noch klarer wäre es, auch das Thallium zu verändern und es gleich Ta (das jetzige Symbol für Tantal) zu setzen und dafür das Symbol für Tantal nach Regel 3 oder 4 zu bilden, also Tn oder auch Tt zu setzen. Dann hießen die 
4 genannten Elemente unzweideutig Thulium $=\mathrm{Tu}$, Thallium $=\mathrm{Ta}$ Thorium $=$ To, Tantal $=$ Tn. Etwas günstiger liegt die Sache für Rhodium $=$ Rh, neben Ruthenium $=\mathrm{Ru}$, Rubidium $=\mathrm{Rb}$, Ra. dium $=$ Ra. . Zwar wäre auch hier Ro geeigneter, doch ist auch Rh unzweideutig. - Es bedarf wohl nur dieser Anregung, um auf der nächsten Tagung der bisherigen internationalen Atomgewichtskommission (von der ja der Deutsche ausgeschlossen werden soll) die genannten Umänderungen, mindestens Thorium $=\mathrm{To}_{0}, \mathrm{zu}$ be wirken

[A. 222.]

\section{Nachtrag zu dem Artikel :}

\section{Wie unsere Heilmittel entstehen. ${ }^{1}$}

Von Prof. Dr. Fritz Hofmann, Breslau.

Herr Dr. R. I a u ch, Berlin, hatte die Freundlichkeit, mich durch Privatbrief darauf aufmerksam zu machen, daß er bereits im Jahre 1893 dem damaligen Pharmakologen der Elberfelder Farbenfabriken Herrn Dr. med. Hermann Hild eb rand t Acetylsalicylsäure zur pharmakologischen Prüfung und Ermittelung ihres eventuellen medizinischen Wertes übergeben hat. Diese Tatsache war mir unbekannt geblieben, obwohl Herr Dr. Hild e b r a n d t darüber berichtet hat ${ }^{2}$ ). Sie hätte bei meinen Ausführungen über den Werdegang des Aspirins natür]ich erwähnt werden müssen. Durch diese Zeilen möchte jch das Versäumte nachholen.

Nachtrag zum Art. 228 (Berichtigung Wöhler betr.). Zu der ,Berichtigung von Herrn Prof. Dr. Wöhler (Angew. Chem. 33, I, 310) erhalten wir folgende Zuschrift:

„Herr Prof. Dr. Hans Goldschmidt verzichtet auf eine Erwiderung, da Herr Prof. Wöhler behauptet hat, er hätte gegen bessere Kenntnis gehandelt."“

\section{Bücherbesprechungen.}

Felix Auerbach, Wörterbuch der Physik. Aus Veits Sammlung wissenschaftlicher Worterbücher. $\mathrm{X}$ und $466 \mathrm{~S}$. Vereinigung wissenschaft]icher Verleger, Berlin und Leipzig 1920. geb. M 26,Die schwierige Aufgabe einer Darstellung des Gesamtgebietes der Physik in Form eines handlichen Wörterbuches hat hier eine ausgezeichnete Lösung gefunden. Die übersichtliche Anordnung nach Stichworten (mehreren tausend) erleichtert ganz außerordentlich das Auffinden eines Gebietes, über das man sich gerade unterrichten will, und auch der Chemiker wird dieses inhaltsreiche Lexikon bald als bequemes Hilfsbuch schätzen lernen.

Die Darstellung ist naturgemäß sehr knapp, aber durchaus klar und, soweit Stichproben ein Urteil ermöglichten, einwandfrei.

Konr. Schaefer. [BB. 116.]

Hager-Mez, Das Mikroskop und seine Anwendung. Handbuch der praktischen Mikroskopie u. Anleitung zu mikroskopischen Untersuchungen von Dr. He r ma n $\mathrm{H}$ a g e r. Nach dessen Tode vollständig umgearbeitet $u$. in Gemeinschaft mit Dr. O. A p p e 1 , Dr. G. Brandes, Dr. P. Lindner, Dr. Th. Lochte neu herausgegeben von Dr. $\mathrm{C}$ a $\mathrm{r} \mathrm{l} \mathrm{M}$ e z. 12. umgearbeitete Aufl. mit 495 Textfiguren. Berlin, Verlag Jul. Springer 1920.

Das Buch liegt nunmehr in seiner 12. Auflage vor. Das sagt eigentlich schon genug. Hervorragende Fachgelehrte haben an der vorliegenden, umgearbeiteten Ausgabe mitgewirkt, und wenn man sagt: Viele Köche verderben den Brei, so straft dieses Buch das alte Sprichwort Lügen und ist im Gegenteil das Produkt besten gemeinschaftlichen Schaffens nach dem Wahlspruch unseres Bruderstaates Österreich: viribus unitis, dem dieser leider nie gefolgt ist. Wir haben in ihm einen wahren Schatz für mikroskopische Untersuchungen zusammengetragenen Wissens. Der erste Teil behandelt um. fassend die Theorie des Mikroskops, seine Einrichtung und praktische Verwendung (Aufhellungs- und Färbemethoden), der zweite Teil behandelt die zu untersuchenden Objekte aus dem nahrungsmittelchemischen und hygienischen Gebiete und dürfte in seiner Reichhaltigkeit praktisch alles umfassen, worüber hier Auskunft verlangt wird. - Das Buch ist unentbehrlich für Untersuchungslaboratorien und hygienische Institute und allen, die mittels des Mikroskops sich die Wunder der Kleinwelt erschließen wollen, ein treuer Lehrer und Freund. von Heygendorff. [BB. 159.]

1) Angew. Chem. 33, I, S. 273-280.

2) Münch. med. Monatsschrift Nr. 1: 1905. H i ld e br a n d t, H e r m a n n, ,Über neuere medikamentös-therapeutische Bestrebungen." Die dort genannte Jahreszahl 1895 muß (nach Dr. L a u c h) 1893 lauten.
Dr. B. Bavink, Finführung in die anorganisehe Chemie. Aus: Naturund Geisteswelt, Verlag von B. G. Teubner, Leipzig und Berlin 1920. Preis M 2,80, geb. M 3,50 und 100\% Teuerungszuschlag. Der Verfasser hat sich die Aufgabe gestellt, in sehr knapper Form die wichtigsten Begriffe der Chemie und ein verhältnismäBig reiches Tatsachenmaterial einem Leserkreis darzustellen, bei dem nicht mehr als Volksschulbildung vorausgesetzt wird. Diese zweifellos recht schwierige Aufgabe hat hier eine sehr anerkennenswerte Bearbeitung gefunden. Der Stoff ist klar geordnet; im ersten Teile werden an der Hand von Beispielen und unter Hinweis auf Experimente, die der Leser selbst anstellen soll, die wichtigsten Grundbegriffe entwickelt. Dann folgen in einem zweiten Teil die Nichtmetalle und der letzte Teil behandelt die wichtigsten Metalle.

Die Fülle der Tatsachen, die auf nur 100 Seiten mitgeteilt werden, ist bedeutend und zwingt den Verfasser zu einer Knappheit der Sprache, daß diese manchmal den Charakter des Telegrammstils annimmt und, trotz aller Klarheit, vom Leser die Gabe einer starken Konzentrationsfähigkeit verlangt. - - Den Veranstaltern von Volkshoch schulkursen sei das Buch für ihre Hörer warm empfohlen; aber auch Studierenden, die Chemie als Nebenfach betreiben, wird diese Einführung nüt:lichere Dienste leisten können, als manches verbreitete? Repetitorium.

Konr. Schaefer. [BB. 122.]

Dr. K. Wolf, Grundriß der Chemie und Mineralogie für die vierte Klasse der Gymnasien und Realgymnasien. Leykam-Verlag, vormals Quidde und Schimpff, Graz 1918. 149 S., 12 Tafeln und 80 Textabb.

geb. M 3,60.

In mancher Beziehung ist dieses, bei billigem Preis gut ausgestattete Schulbuch vielen seiner Artgenossen überlegen. Es vermeidet die planlose Überfülle chemischer Einzelheiten, welche für den Schulunterricht unwesentlich oder schädlich sind. Es zeichnet sich durch die Klarheit und Schönheit vieler Abbildungen (z. B. die mustergültige Photographie einer Bessemeranlage) aus. Andererseits ist es auch von weitverbreiteten Mängeln der meisten Schul-Chemiebücher nicht frei (z. B. veraltete statistische Angaben). Der Mineralogie und Krystallographie räumt es sehr viel Platz ein. Alfred Stock. [BB. 167.$]$

Chemie für Techniker. Leitfaden für Maschinen- und Bautechniker von Dr. $\mathrm{O}$ s k a r S c h mid t. 8. Aufl. Mit 54 Figuren im Text. VI und 170 S. Verlag von Konrad Wittwer, Stuttgart 1920. Preis M 7,50.

Der Leitfaden sucht unter weitgehender Einschränkung des theoretischen Teils und selbst unter Weglassung von Formeln, wo sie dem Verfasser entbehrlich erscheinen (z. B. bei Borax) dem angehenden Techniker alle chemischen Vorgänge verständlich $\mathbf{z u}$ machen die ihm in seiner Praxis begegnen können. Diese Einschränkung durfte aber nicht so weit gehen, chemisch gebundenes und Krystallwasser einander gleichzusetzen (S. 117). Hingegen ist es überflüssig, Molekularformeln in Klammern einzuschließen, wie es stellenweise geschieht, während andererseits in den Formeln krystallwasserhaltiger Verbindungen das Krystallwasser von der Formel der Verbindung entweder durch Komma oder (besser) durch Pluszeichen abzutrennen wäre. - Durch Anwendung eines kleineren Druckes für einen Teil der technisch wichtigen Abschnitte ist es gelungen, alles für die Erörterung zu erfassen, was sowohl den Bau. techniker wie den Maschinentechniker angeht, wenn sich auch nicht leugnen läßt, daß beide in getrennt für sie geschriebenen Lehr. büchern noch ausführlicher hätten belehrt werden können. - Der Satz, daß Calciumverbindungen in den meisten Böden so reichlich vorhanden seien, daß eine ,Kalkdüngung" entbehrlich ist (S. 93), wird kaum die Zustimmung aller Agrikulturchemiker finden. Ferner trifft es leider nicht zu, daß wasserlösliche Salze aus Tonen durch Auswintern und Einsumpfen (S. 119) möglichst entfernt werden können. Doch sind das nur kleine Ausstellungen für eine spätere Neuauflage, die gegenüber der Tatsache wenig ausmachen, daß in dem Buche nichts Wesentliches fortgelassen ist. Im Verhältnisse zum Umfange des Buches ist das Gebotene vielmehr als $\mathrm{sehr}$ reich h a l t ig zu bezeichnen, so daß der Leitfaden einem guten, durch Experimente unterstiutzten Unterrichte zugrunde gelegt werden kann. Jedem chemisch bereits unterrichteten Techniker wird es auch als Nachschlagebuch gute Dienste leisten. $\quad$ Dr. Rudolf. [BB. 174.]

Technisehe Chemie für Masehinenbauschulen. Von Prof. Dr. S i e g fried Jakobi. 2. Aufl. Mit $100 \mathrm{Abb}$. im Text. VIII. und 160 S. Verlag von Julius Springer, Berlin 1920. Preis M 11,-

Dadurch, daß sich das Buch ausschließlich an Maschinen- und Elektrotechniker wendet, ist es gelungen, diesen eine gute und ge. nügend ausführliche Darstellung der ihnen im Berufe vorkommenden chemischen Begriffe und Vorgänge zu geben. Es stellt keine leichte Aufgabe dar, Schüler oder Studierende, für die Chemie nur ein "Nebenfach" ist, in die theoretischen Grundlagen unserer Wissenschaft einzuführen. Man merkt dies auch allen für solche Zwecke geschriebenen Lehrbüchern an. Dem vorliegenden aber ist durch die bestehenden Vorschriften betreffs des Lehrplans an den preußischen Maschinenbauschulen eine gewisse Beschränkung der theoretischen Erörterungen auferlegt gewesen. Wieweit sich diese den Fragen 